\title{
Visceral fat measured by computed tomography and the risk of breast cancer
}

\author{
Myeong Seong Kim ${ }^{1,2}$, Yoon-Jung Choi ${ }^{3}$, Yoon Hyeon Lee ${ }^{1}$ \\ ${ }^{1}$ Department of Public Health Administration, Namseoul University, Cheonan, South Korea; ${ }^{2}$ Department of Radiology, National Cancer Center, \\ Goyang-si, South Korea; ${ }^{3}$ Department of Preventive Medicine, Seoul National University College of Medicine, Seoul, South Korea \\ Contributions: (I) Conception and design: MS Kim, YH Lee; (II) Administrative support: YH Lee; (III) Provision of study materials or patients: MS \\ Kim, YJ Choi; (IV) Collection and assembly of data: MS Kim; (V) Data analysis and interpretation: MS Kim, YJ Choi; (VI) Manuscript writing: All \\ authors; (VII) Final approval of manuscript: All authors. \\ Correspondence to: Yoon Hyeon Lee, PhD, Professor. Public health administration, Namseoul University, 91 Daehak-ro, Seonghwan-eup, Seobuk-gu, \\ Cheonan, Chungcheongnam-do, South Korea. Email: skylee@nsu.ac.kr.
}

Background: Obesity is a risk factor for the development of breast cancer, which can be examined to assess the association between abdominal fat distribution through computed tomography (CT) and risk of breast cancer by hormone receptor (HR) status in women.

Methods: We examined 234 cases of newly diagnosed breast cancer patients and 211 controls consisting of health examinees in the Republic of Korea. The abdominal body fat distribution was measured by CT scan. The subjects' medical information, obtained from electronic medical records (EMR), were analyzed to verify the association between visceral fat and the type of breast cancer by HRs.

Results: While visceral obesity increased the risk of breast cancer in post-menopausal women by 1.50 (95\% CI, 0.75-2.98), the effect was not observed in pre-menopausal women 0.86 (95\% CI, 0.44-1.62). Also, histological grade was associated with visceral fat, which higher histological grade score $(\geq 2)$ showed a slightly increased mortality rate $(\mathrm{r}=0.15, \mathrm{P}=0.07)$. Patients with negative progesterone receptors tended to have a higher abdominal fat ratio than patients with positive progesterone receptors. Especially in premenopausal women, higher distribution of abdominal fat ratio in breast cancer patients had somewhat more of an effect on negative than positive HRs [estrogen receptor (ER), $\mathrm{P}=0.12$; progesterone receptor $(\mathrm{PR})$, $\mathrm{P}=0.06$; human epidermal growth factor receptor 2 (HER2), $\mathrm{P}=0.43$ ].

Conclusions: Our results suggest that the clear association between breast cancer and distribution of abdominal fat, and the abdominal fat ratio, are useful indicators for risk factors for breast cancer as well as malignancy.

Keywords: Breast cancer; intra-abdominal fat; computed tomography (CT); obesity

Submitted Feb 01, 2019. Accepted for publication Aug 29, 2019.

doi: $10.21037 /$ tcr.2019.09.16

View this article at: http://dx.doi.org/10.21037/tcr.2019.09.16

\section{Introduction}

Breast cancer has the highest incidence rate among all cancers in women, and over 411,000 deaths result from breast cancer annually (1). Although South Korea has relatively low breast cancer incidence rates compared to developed countries, breast cancer is the most common cancer among women in Korea (except for thyroid cancer), and the incidence rate is recently rapidly rising, increasing from 43.8 (per 100,000 women-years) in 2009 to 58.4 (per $1,000,000$ women-years) in 2016 (Korean National Cancer Information Center 2019) (2). The incidence rate of breast cancer has increased in South Korea due to the intake of a high-fat Western diet, lack of physical activity, obesity, and 
rising stress levels, which are the main contributory factors (1). Many epidemiological studies indicate that obesity contributes to the increased incidence rates of cancer, and have assessed that $15-20 \%$ of all cancer deaths in the Unites States are from obesity (3). According to previous studies, mortality is also higher in post-menopausal breast cancer patients with a body mass index (BMI) $\geq 30.0$ (obesity), two to six times higher than those with a BMI $<25$ (normal) (4). However, in pre-menopausal women, $\mathrm{BMI}$ and breast cancer mortality were negatively associated $(4,5)$. Previous studies have shown associations among body fat distribution, hormone levels, and metabolic markers in women depending on BMIs, and suggested that body fat distribution may play a role in the risk of breast cancer (6). Also, obesity in breast cancer patients is associated with both reduced a survival rate and increased recurrence rate, irrespective of menopausal status, even after adjustment for clinical stages and therapy (7).

Unlike the general perception that abdominal obesity is defined by waist circumference (WC) and BMI measurement, these do not ideally reflect the amounts and the sites of deposition of the adipose tissue compared to measurements obtained from computed tomography (CT), which is considered the gold-standard method for determining the quantity of abdominal fat (8). In general, components of abdominal adiposity are divided into the subcutaneous adipose tissue (SAT) and visceral adipose tissue (VAT) areas. The content of VAT has variations among age, sex, and race (9), and is more metabolically active than SAT since it has high lipolytic activity and releases large amounts of free fatty acids $(10,11)$. Thus, optimal measurement of adipose tissue would be necessary for both the amount and the site of deposition of the adipose tissue (12).

Most previous studies on the relationship between obesity and breast cancer were based on BMI or WC measurements (4-6). This study, on the other hand, demonstrates a more accurate relationship between breast cancer incidence and obesity by measuring the amount of quantitative adipose tissue, excluding subcutaneous fat through CT. Thus, the purpose of this study was to conduct a case-control study to assess the association between abdominal fat distribution through CT images and the risk of breast cancer in pre-menopausal and post-menopausal women. Also, we investigated the relationship between abdominal fat distribution and characteristics of breast cancer according to hormone receptor (HR) status.

\section{Methods}

\section{Study design and subjects}

The institutional review board approval for the study was obtained from the local Research Ethics Committee on July 30, 2014, and written informed consent was obtained from all participants (NCC2014-0124). The selected patient criteria were those who visited the Breast Cancer Center in the National Cancer Center (NCC) in South Korea during the study period and were newly diagnosed with breast cancer; we additionally excluded patients who received surgery, radiation therapy, and chemotherapy in any other hospitals. The control group consisted of healthy participants without a history of cancer who went through a health examination from the National Health Insurance Services and the private medical health check-up in the NCC from July $30^{\text {th }}, 2014$ to September $30^{\text {th }}, 2015$.

\section{Participant information}

Variations in a woman's reproductive history may be indicative of the risk for developing breast cancer. To determine whether menstruation affects breast cancer occurrence, study participants completed questionnaires on menopausal status, age at menarche, parity, and breastfeeding.

All patient information was obtained from electronic medical records (EMR) at the NCC hospital, including factors such as height, weight, histological grade of breast cancer, lymph node involvement, the status of estrogen receptor (ER), the progesterone receptor $(\mathrm{PR})$, and the human epidermal growth factor receptor 2 (HER2). Each subject's weight and height were gathered from the nurses' information sheets registered at each admission interview to calculate BMI. BMI $\left(\mathrm{kg} / \mathrm{m}^{2}\right)$ was calculated as body weight in kilograms divided by height in meters squared. In addition to the patients' histologic grade and lymphatic invasion, data was established post-operatively by the surgeon and at the ER and PR levels obtained from the pathology report in the EMR.

The specimens were evaluated according to the following histopathologic features: histological type of carcinoma, black nuclear grade (nuclear grade 1, poorly differentiated; grade 2, moderately differentiated; and grade 3, well differentiated), and modified Bloom-Richardson histological grade (histological grade 1, well differentiated; grade 2, moderately differentiated; and grade 3, poorly 

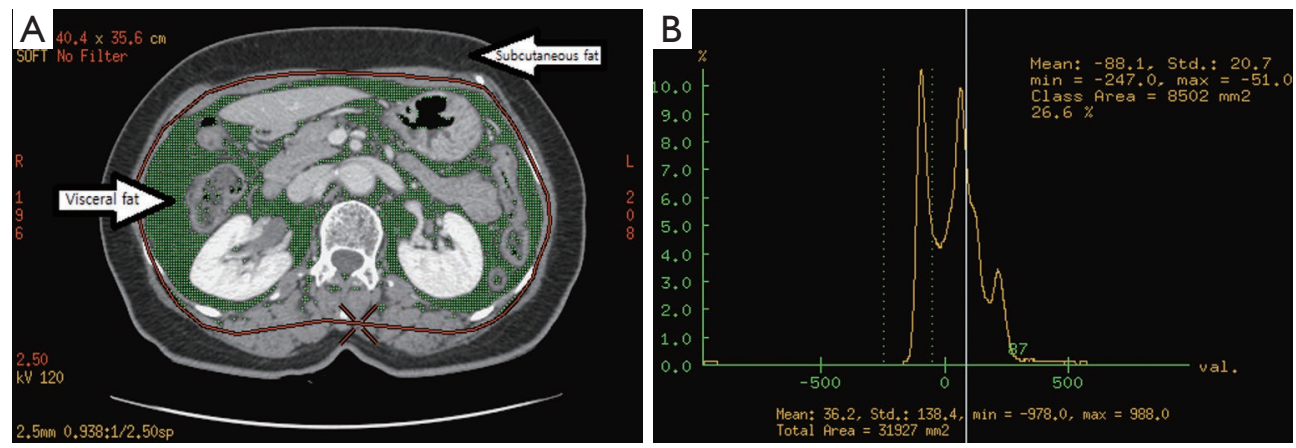

Figure 1 Measurements of Abdominal Fat from a CT Image. (A) Visceral adipose tissue (shaded) within the intra-abdomen and total adipose tissue outside the abdomen; (B) histogram of CT numbers obtained from an umbilicus level image. The maximum peak represents fat, and the smaller peak represents lean tissue. The admissible range of CT numbers classified as fat was -50 to -250 . CT, computed tomography.

differentiated). For dichotomous-dependent variables, nuclear grade was classified as high (grade 1) versus low (grades 2 and 3), and histologic grade as low (grades 1 and 2) versus high (grade 3). Expressions of ER, PR, and HER2 were evaluated using standard avidin-biotin complex immune histochemical staining methods. The ER and PR statuses were assessed using the Allred scoring, which was expressed as the sum of the proportion score and the intensity score of positively stained tumor cells. Tumors with an Allred score of at least 3 were regarded as positive, and the others were regarded as negative. The intensity of HER2 staining was scored as $0,1+, 2+$, or $3+$. Tumors with a $3+$ score were classified as HER 2 positive, and tumors with a 0 or $1+$ score were classified as negative. In tumors with a $2+$ score, gene amplification via fluorescence in situ hybridization was used to determine HER2 status.

\section{Measurement of abdominal fat}

All CT examinations were performed on the 64-Multi detector row computed tomography system (Lightspeed VCT and Discovery HD 750; GE Healthcare system, Milwaukee, WI, USA). CT exposure parameters were $120 \mathrm{kVp}$, auto exposure control mA (mA range, 100-350), and pitch 1:1. Imaging data were reconstructed every $10 \mathrm{~mm}$.

We measured abdominal fat with a CT scan at the level of umbilicus, or the fourth lumbar vertebra, which is a valid method to measure abdominal fat in humans (13). To quantify the subject's adipose tissue, all CT data were transferred to a commercially available workstation computer (Advantage Windows Workstation, version 4.5, GE Healthcare), and then we measured the total adipose tissue (TAT) volume and VAT (Figure 1A) with CT numbers from -50 and -250 Hounsfield Units (HU) (Figure 1B), which correspond to the CT histogram of adipose tissue at the umbilicus level.

All visceral fat measurements were taken by a single radiology technician throughout the study. To minimize measurements error by a single person, measurements of abdominal fat were performed by another technician in samples randomly assigned by using a double-blind random allocation (blank participant information) with permuted duplication data assignments.

SAT and fat ratio were calculated using the following equations:

$$
\begin{aligned}
& \mathrm{SAT}=\mathrm{TAT}-\mathrm{VAT} \\
& \text { Fat ratio } \%=(\mathrm{VAT} / \mathrm{TAT}) \times 100
\end{aligned}
$$

To minimize measurement error by one person, we randomly divided the case and control samples by 30 participants each (by SPSS random selection procedures). Intrapersonal differences between Test 1 and Test 2 were subsequently tested separately, measured by two trained radiology technicians using only identifier numbers in the measurement computer (AW4.5). To compare the intrapersonal difference in measurement of visceral fat, we performed correlation and a Chi-square test. The value of correlation ( $\mathrm{r}$ ) and Chi-square (P) between the two measurements represented a similar pattern in each case and control (VAT: $r=0.98, P=0.24$; fat ratio: $r=0.96, P=0.23$ in cases; VAT: $r=0.98, \mathrm{P}=0.23$; fat ratio: $\mathrm{r}=0.99, \mathrm{P}=0.23$ in controls).

\section{Statistical analysis}

We analyzed the collected data to evaluate the characteristics 
of cases and controls. All means and distributions of demographic and characteristics were examined using the $t$-tests and chi-square tests. Multiple regression analysis was conducted to determine the relationships of the many variables with breast cancer after using the normally transformed variates. Among the correlated variates, odds ratios and $95 \%$ CIs were analyzed by entered logistic regression. Statistical analysis was performed using SPSS Statistics v. 20 (SPSS Inc., Chicago, IL, USA), and P values $<0.05$ were considered statistically significant.

\section{Results}

\section{General distribution of subjects}

The total number of patients was 234, ranging from 32 to 82 years old, with a mean age of 52.6 years old, and the 211 control participants ranged from 27 to 62 years, with a mean age of 52.3 years old. Cases and controls were matched for age ( \pm 2 years).

The randomly selected case participants had a mean abdominal fat ratio of $36.9 \%$ with a mean age of 56.8 years old, and the control participants had a mean abdominal fat ratio of $32.4 \%$ with a mean age of 53.8 years old. The comparison of the two duplicate samples, with an almost perfectly matched scatter plot, demonstrates the validation of the abdominal fat measurement.

Table 1 shows general distribution of all subjects' sociodemographic characteristics.

Table 2 shows the general distribution of abdominal fat among the subjects. Anthropometric factors were divided into the tertiles, and we performed logistic regression to evaluate association between anthropometric measurements and the risk of breast cancer. In all subjects (no distinction between pre-and post-menopausal women for analysis), the multivariable adjusted odds ratio of incidences of breast cancer for the highest $v s$. the lowest tertiles of abdominal fat ratio and visceral fat were $1.34(\mathrm{P}=0.25,95 \% \mathrm{CI}, 0.82-2.16)$ and $1.51(\mathrm{P}=0.20,95 \% \mathrm{CI}, 0.91-2.51)$, respectively. Thus, the association between visceral fat and incidence of breast cancer in all subjects is not statistically significant.

\section{Association between abdominal fat and breast cancer classified as menopausal status}

Associations between measured abdominal fat and breast cancer were different according to menopausal status, but they were not statistically significant (Table 3). The multivariable-adjusted odds ratio of breast cancer for the highest $v s$. the lowest tertiles of abdominal fat ratio were 0.88 (95\% CI, $0.46-1.67 ; \mathrm{P}=0.6$ ) in pre-menopausal women and 1.53 (95\% CI, 0.73-2.88; $\mathrm{P}=0.04$ ) in post-menopausal women. Although statistically not significant, breast cancer was positively associated with VAT in post-menopausal women, but not in pre-menopausal women. Abdominal SAT, the multivariable-adjusted odds ratio of breast cancer for the highest $v s$. the lowest tertiles of pre-menopausal women were 0.53 (95\% CI, 0.22-1.04; $\mathrm{P}=0.29)$, the lowest inverse associated with breast cancer incidence.

\section{Association between HR and obesity for breast cancer}

Figure 2 and Table 4 show the association between HR status and body fat related anthropometric factors. Generally, patients with negative receptors had higher abdominal fat ratios than patients with positive receptors (ER, $\mathrm{P}=0.12 ; \mathrm{PR}, \mathrm{P}=0.06$; HER2, $\mathrm{P}=0.43$ ). The HER2 score was associated with greater abdominal fat ratio in pre-menopausal women (ER, $\mathrm{P}=0.41 ; \mathrm{PR}, \mathrm{P}=0.33$; HER2, $\mathrm{P}=0.02$ ), but not in post-menopausal women. Thus, abdominal fat was associated with HER2 negative breast cancer in pre-menopausal women, but not in postmenopausal women. However, BMI did not show such a pattern as in hormonal receptors.

Similar to Table 4, crude odds ratios of breast cancer with receptor according to abdominal fat distribution show that increased VAT was positively associated with negative hormone status in breast cancer patients. The odds ratio values of ER, PR, and HER2 statuses for the highest vs. the lowest median of abdominal fat ratios and VAT were as follows. For ER status, 1.31 (95\% CI, 0.74-2.29) and 1.42 (95\% CI, 0.81-2.48); for PR status, 2.03 (95\% CI, $1.19-3.47)$ and 1.93 (95\% CI, 1.12-3.33); and for HER2 status, 1.46 (95\% CI, 0.83-2.58) and 1.59 (95\% CI, 0.902.80 ), respectively. Thus, generally higher distributions of abdominal fat ratio or VAT were associated with negative receptors, but not with positive receptors in breast cancer.

Figure 3 shows the effect of VAT and BMI on histologic grade of breast cancer. Higher VAT was positively related with histologic grade $(\beta=1.97, \mathrm{P}=0.001$ for regression; $\mathrm{r}=0.15, \mathrm{P}=0.07$ for Pearson correlation), but with weak statistical power. Unlike the results of correlation with VAT for histologic grade, BMI showed low correlation with histologic grade $(\beta=1.20, \mathrm{P}=0.07$ for regression; $r=0.01$, $\mathrm{P}=0.79$ for Pearson correlation). 
Table 1 Distribution of all subjects' socio-demographic characteristics

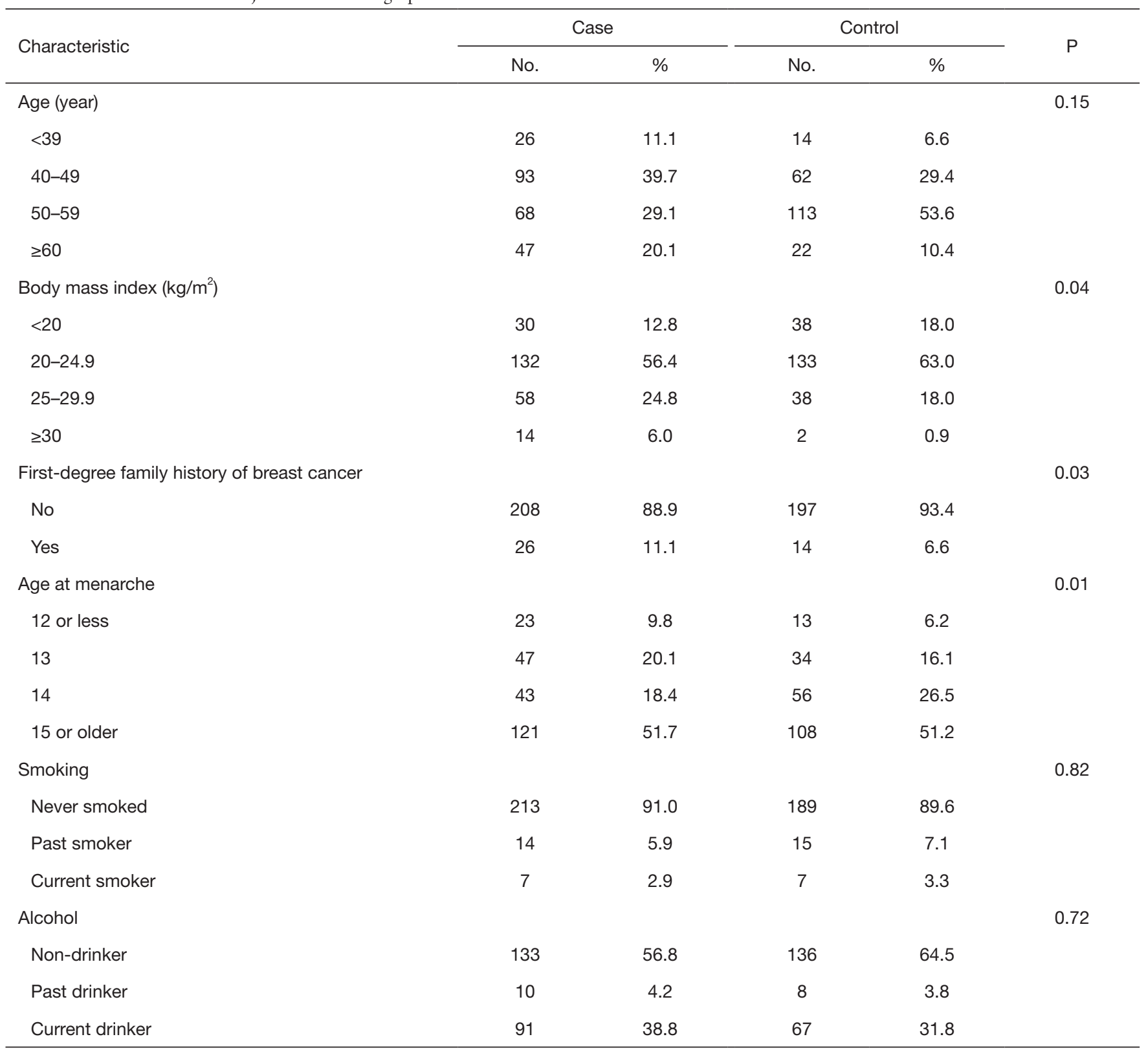

Results were statistically analyzed with a Chi-square test.

\section{Discussion}

This study provides new insight into the association between breast cancer risk and the distribution of abdominal fat (VAT) using a CT technique. Obesity has been indicated as a risk factor for breast cancer in post-menopausal women (12-14). While obesity may increase the risk of breast cancer in post-menopausal women, previous studies have shown the protective effects of obesity in pre-menopausal women, but the mechanisms for the negative association are poorly understood $(13,14)$. Different from the previous study related with obesity's effect on breast cancer, BMI showed a positive association with breast cancer in both pre- and post-menopausal women $(6,15)$. The underlying mechanisms are an unclear incidence of breast cancer according to menopause status, but may be attributed to the menstruation cycle by sex hormone concentrations 
Table 2 Distribution of abdominal fat in all subjects

\begin{tabular}{|c|c|c|c|}
\hline Subjects & Control mean \pm SD & Case mean $\pm S D$ & $\mathrm{P}$ \\
\hline \multicolumn{4}{|c|}{ Abdominal total fat $\left(\mathrm{mm}^{2}\right)$} \\
\hline Total & $23,403.0( \pm 8,704.3)$ & $23,279.1( \pm 10,068.2)$ & 0.96 \\
\hline Pre-menopause & $21,143.3( \pm 7,716.2)$ & $19,476.5( \pm 9,324.0)$ & 0.17 \\
\hline \multicolumn{4}{|c|}{ Abdominal visceral fat $\left(\mathrm{mm}^{2}\right)$} \\
\hline Total & $7,992.6( \pm 4,549.6)$ & $8,186.7( \pm 5,424.8)$ & 0.64 \\
\hline Pre-menopause & $6,725.8( \pm 3,862.5)$ & $6,151.1( \pm 4,604.1)$ & 0.34 \\
\hline Post-menopause & $9,091.3( \pm 4,823.7)$ & $1,0781.1( \pm 5,301.1)$ & 0.60 \\
\hline Pre-menopause & $14,417.5( \pm 4,794.5)$ & $13,325.4( \pm 5,432.5)$ & 0.13 \\
\hline Post-menopause & $16,271.3( \pm 5,479.0)$ & $17,344.6( \pm 5,027.1)$ & 0.89 \\
\hline \multicolumn{4}{|c|}{ Abdominal fat ratio (\%) } \\
\hline Total & $32.6( \pm 8.80)$ & $32.6( \pm 10.55)$ & 0.91 \\
\hline Pre-menopause & $30.92( \pm 8.0)$ & $29.3( \pm 9.65)$ & 0.22 \\
\hline Post-menopause & $34.19( \pm 9.24)$ & $36.8( \pm 10.1)$ & 0.19 \\
\hline
\end{tabular}

Results were statistically analyzed with a $t$-test (2 tailed).

and abnormality at a particular time within the patterns of the follicular phase of the cycle $(6,15,16)$. Characteristics of breast cancer are different in post-menopausal and premenopausal women due to a whole spectrum of biological differences. Breast cancer in pre-menopausal women presented a higher prevalence of hormone independent, more aggressive tumors and poorer prognosis compared to breast cancer in post-menopausal women $(17,18)$.

Generally adipose tissue is composed of subcutaneous and visceral tissue; SAT is defined as the layer of subcutaneous tissue, whereas VAT is within the main cavities of the abdomen, known as organ fat or intra-abdominal fat. VAT is more metabolically active and susceptible to hormonal changes than SAT. It is correlated with insulin action (hyperinsulinemia and insulin resistance), increased levels of free fatty acids, decreased level of sex hormone binding globulin levels, and elevated bioavailability of estradiol (19), which may play a role related to increased breast cancer risk, although SAT may play a similar role $(10,16)$. Generally, similar age, gender, and BMI have the same percentage of body fat distribution. However, despite a similar BMI or
WC, VAT content shows variations in age, sex, and race (9). The assessment of fat distribution is an important issue in obesity research because this VAT seems to be most strongly associated with not only metabolic disorders but also cancer $(11,19)$. However, using CT is an accurate method for measuring body fat distribution due to the offered insight into cross-sectional body images, including the quantification of body fat (20). Ideally, measurement of the abdominal fat compartment on breast cancer risk should rely on techniques such as CT, which provides the best overall predictor of breast cancer $(20,21)$.

VAT is linked to the development of breast cancer resulting from higher levels of estrogen derived from aromatization of androstenedione within the larger fat stores (15). Thus, VAT is usually positively correlated with the levels of free steroid hormones (22).

On the other hand, identifying HR status of invasive breast cancer is known to be useful as a prognostic and metabolic factor, and it has become standard practice in the treatment of breast cancer (23). Also, in this study, ERnegative, PR-negative, and HER2-negative breast cancers 
Table 3 Odds ratios and $95 \%$ CI on breast cancer risk regarding distribution of abdominal fat, pre- and post-menopausal status in all subjects



*, odds ratio adjusted for age (year), menstruation (post-menopausal; pre-menopausal), family history (positive family history; negative family history), and age at menarche (12 year or less; 13 year; 14 year; 15 year or older).

showed markers of rapid growth, a reflection of their poor biological character $(18,24)$. We used a classification system and logistic regression approach to analyze the association between abdominal distribution fat and hormone status. Our findings show that higher abdominal distribution of fat (VAT) was correlated with elevated odds ratio for ER-negative, PR-negative, and HER2-negative breast cancers with negative status in breast cancer patients. Also, abdominal visceral fat was more associated with the risk of ER-negative, PR-negative, and HER2-negative breast 

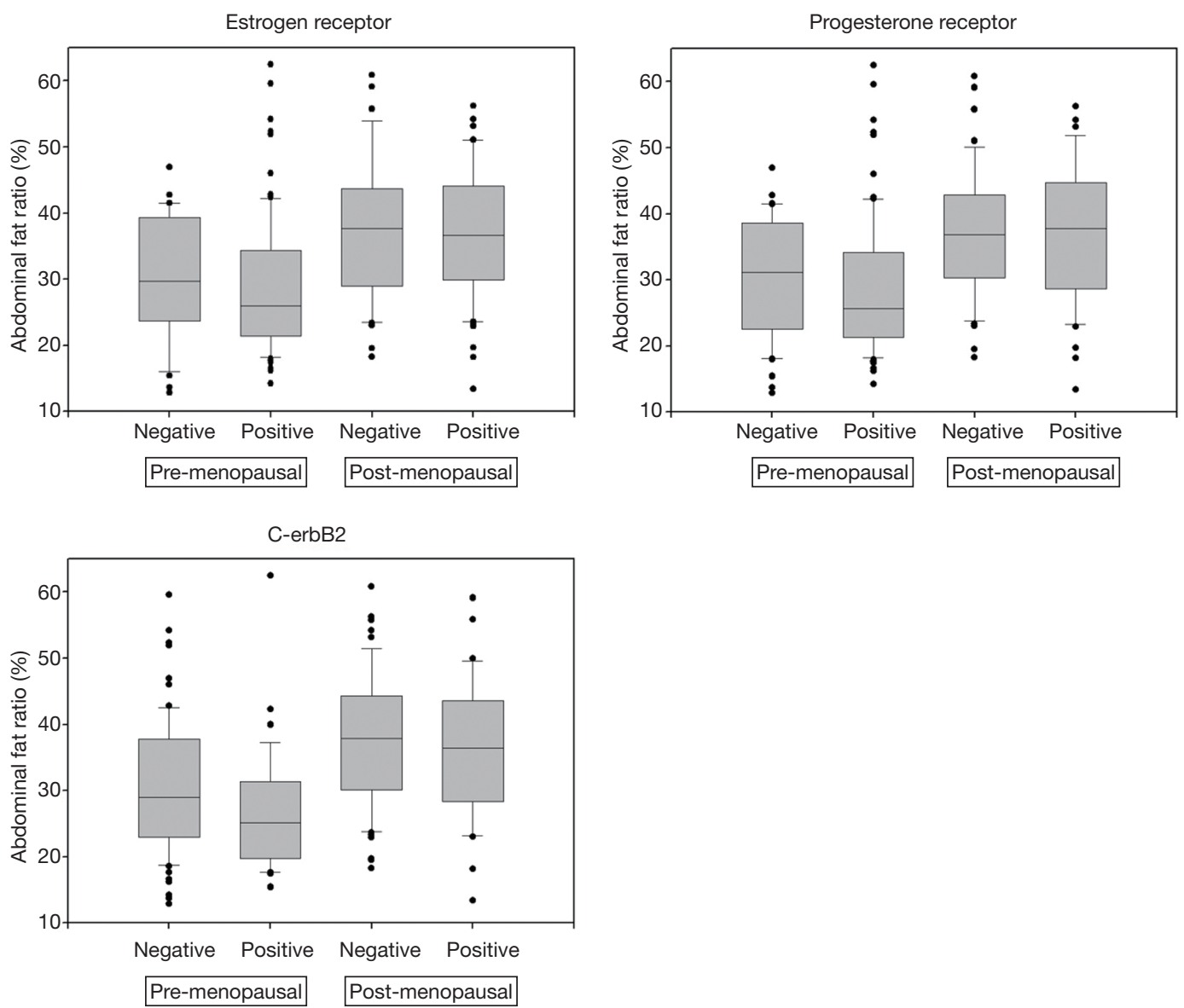

Figure 2 Abdominal fat ratio and hormone status diagram in breast cancer patients. Generally negative hormone patients had a higher abdominal fat ratio than positive hormone patients in only breast cancer patients. (A) Diagram of abdominal fat ratio and classified estrogen receptor score according to menopause status; (B) diagram of abdominal fat ratio and classified progesterone receptor score according to menopause status; (C) diagram of abdominal fat ratio and classified Herceptin score according to menopause status. Dots represent outliers.

cancer than with the risk of ER-positive, PR-positive, and HER2-positive breast cancer, which suggests that visceral fat may influence the risk of breast cancer, especially in pre-menopausal women. In general, breast cancer patients with ER-positive, PR-positive, and HER2-positive breast cancer are at lower risks of mortality after their diagnosis compared to each of ER-negative, PR-negative, HER2negative breast cancer $(25,26)$. Further, hormonal therapy is suitable for patients diagnosed ER-positive, PR-positive, and HER2-positive breast cancer, but patients with HRnegative breast cancer are not suitable for hormone therapy (6). Specifically, HER2 negative cancers are more likely to be poorly differentiated, of higher histological grade, associated with a higher recurrence rate, and lack the benefit of specific target therapy, such as trastuzumab (26). Higher VAT is related with a worse prognosis and poor treatment outcome in breast cancer, while hormone positivity in breast cancer is related to a good prognosis (17). Therefore, treatments options for these triple-negative breast cancer patients, classified as ductal carcinoma, are more limited. Furthermore, the mortality rate of women with negative hormone tumors reaches a peak level 2 to 4 years after diagnosis, while women with HR-positive breast cancers show a consistent long-term survival rate (27).

According to our results, regardless of menopausal status, obese breast cancer patients are more likely to have a poor prognosis than those who are not obese (28-30). That is, the effects (VAT has a poor outcome) are similar in both pre- and post-menopausal women (28-30). Also, our data on the association between histologic grade and distribution of abdominal fat support the hypothesis that the effects of VAT on breast cancer risk are different according to HR 
Table 4 Comparison of average anthropometric factors (distribution of abdominal fat and BMI) and classified by hormone in only breast cancer patients

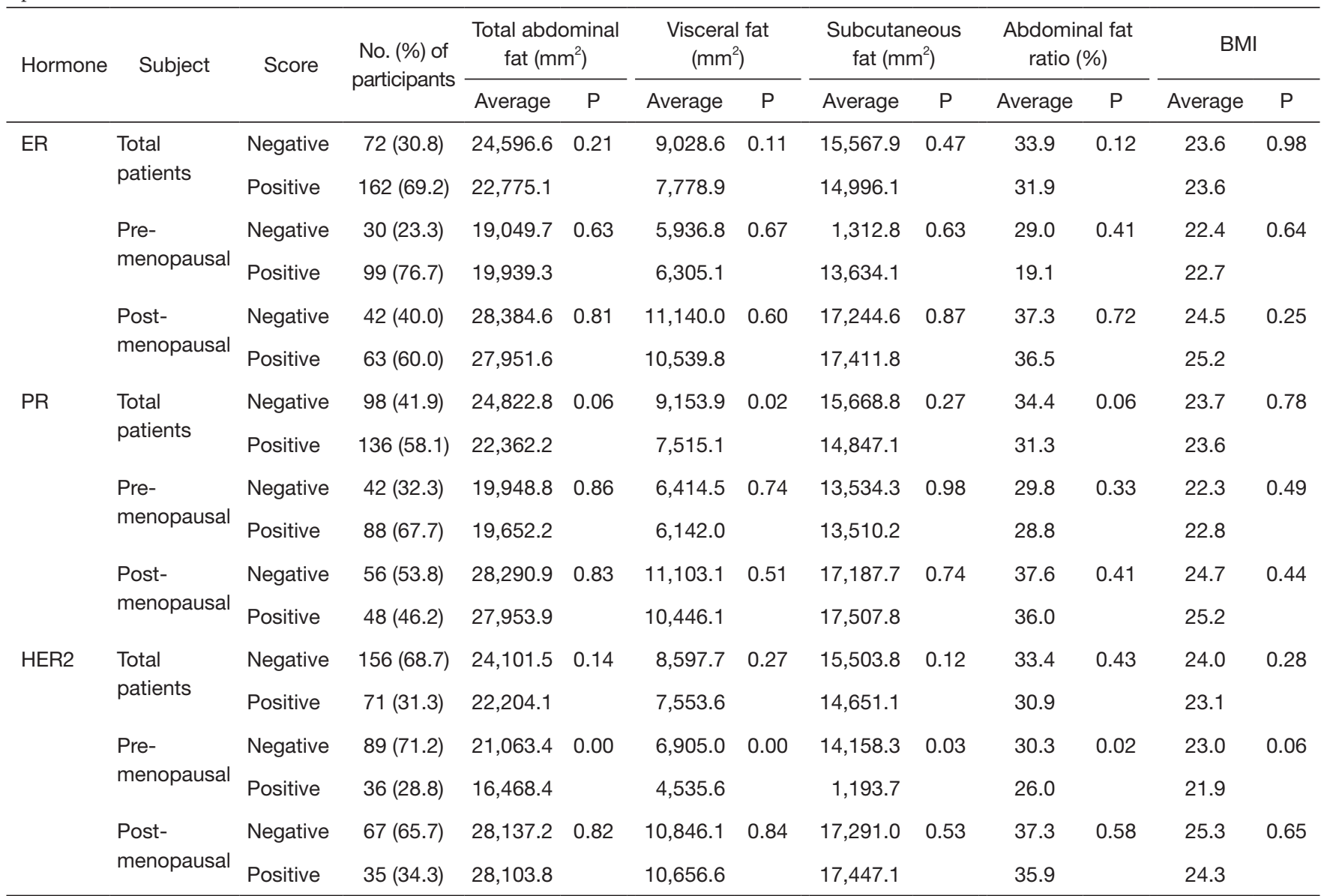

ER and PR were classified as Allred score: $\geq 3=$ positive, $0-2=$ negative; HER2 was classified: $\geq 3=$ positive, $0-1=$ negative; P: $t$-test (2-tailed). BMI, body mass index; ER, estrogen receptor; PR, progesterone receptor; HER2, human epidermal growth factor receptor 2.

A

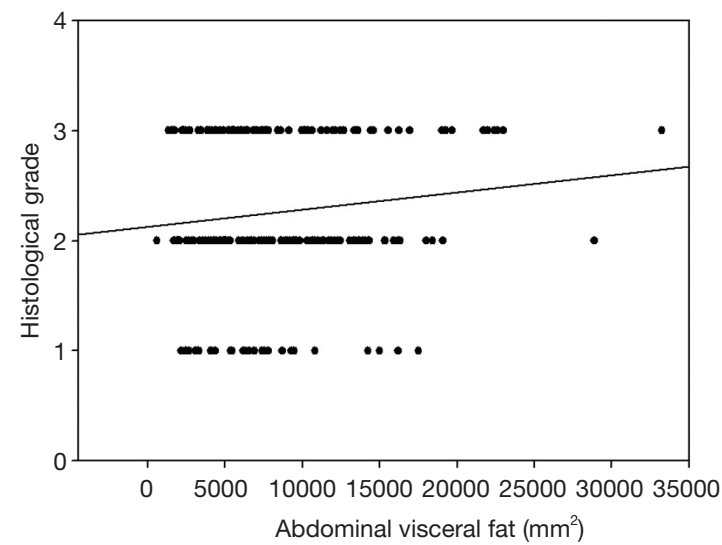

B

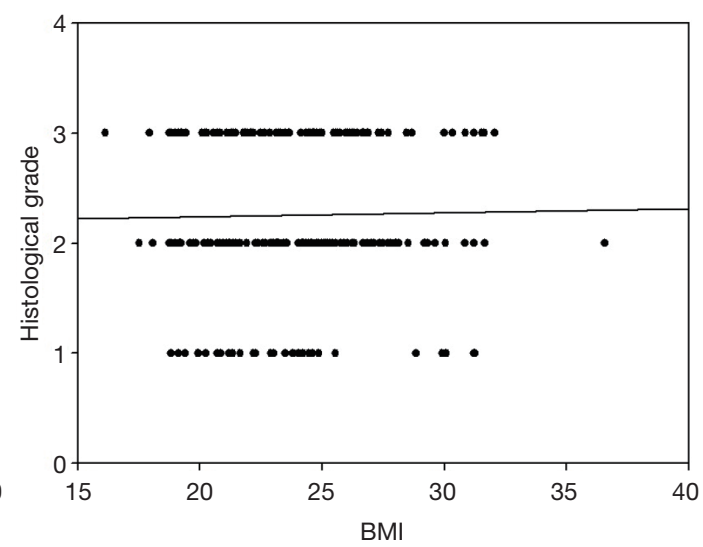

Figure 3 A simple scatter-regression graph, abdominal visceral fat or BMI effect on histological grade of breast cancer in all breast cancer patients. (A) Correlation between abdominal visceral fat and histological grade. A positive relation shows with histological grade; (B) correlation between BMI and histological grade. No relation shows with BMI. BMI, body mass index. 
status. And previous studies have found a strong correlation between the histologic grade and hormonal receptor status according to distinct gene expression profiles (28). However, BMI showed no association with histologic grade in any HR. Though ER status itself is known as a risk factor for breast cancer, our analysis was restricted to breast cancer patients because regular participants were not included in the health examination service by the national health insurance.

There are many contributory factors on the risk of breast cancer, but this study has a limitation because the factors for incidence of breast cancer were assessed by association with only the amount of abdominal fat. In order to obtain reliable related to breast cancer and obesity studies, more institutes and more subjects are needed.

Korea achieved high economic development in the past 30 years, and the lifestyle and dietary habits of the Korean people have shown a clear shift to the westernized style compared to previous generations. In addition, related previous studies have indicated that obesity contributes to an increased incidence of breast cancer. Most previous studies related to obesity and cancer used BMI and WC as biomarkers. However, in this paper, it is significant that we investigated the association between breast cancer and obesity by measuring the amount of VAT, which adversely affects health. In addition, further studies are to be conducted; we believe that visceral fat can be used as a biomarker for breast cancer.

\section{Conclusions}

In conclusion, incidence of breast cancer has a positive correlation with the amount of VAT, especially in postmenopausal women. Also, greater VAT is associated with the negative hormone, which makes the hormone treatment of breast cancer difficult.

\section{Acknowledgments}

Funding: This work was supported by the Research Project for Improving Quality in Namseoul University in the Republic of Korea.

\section{Footnote}

Conflicts of Interest: All authors have completed the ICMJE uniform disclosure form (available at http://dx.doi. org/10.21037/tcr.2019.09.16). The authors have no conflicts of interest to declare.

Ethical Statement: The authors are accountable for all aspects of the work in ensuring that questions related to the accuracy or integrity of any part of the work are appropriately investigated and resolved. This study was conducted in accordance with the Declaration of Helsinki (as revised in 2013). The institutional review board approval for the study was obtained from the local Research Ethics Committee, and written informed consent was obtained from all participants (NCC2014-0124).

Open Access Statement: This is an Open Access article distributed in accordance with the Creative Commons Attribution-NonCommercial-NoDerivs 4.0 International License (CC BY-NC-ND 4.0), which permits the noncommercial replication and distribution of the article with the strict proviso that no changes or edits are made and the original work is properly cited (including links to both the formal publication through the relevant DOI and the license). See: https://creativecommons.org/licenses/by-nc-nd/4.0/.

\section{References}

1. McGuire S. World Cancer Report 2014. Geneva, Switzerland: World Health Organization, International Agency for Research on Cancer, WHO Press, 2015. Adv Nutr 2016;7:418-9.

2. Jung KW, Won YJ, Kong HJ, et al. Prediction of Cancer Incidence and Mortality in Korea. Cancer Res Treat 2019;51:431-7.

3. Jiralerspong S, Goodwin PJ. Obesity and breast cancer prognosis: evidence, challenges, and opportunities. J Clin Oncol 2016;34:4203-16.

4. Gathirua-Mwangi WG, Zollinger TW, Murage MJ, et al. Adult BMI change and risk of breast cancer: National Health and Nutrition Examination Survey. Breast Cancer 2015;22:648-56.

5. Schoemaker MJ, Nichols HB, Wright LB, et al. Association of body mass index and age wit subsequent breast cancer risk in premenopausal women. JAMA Oncol 2018;4:e181771.

6. Harris HR, Willett WC, Terry KL, et al. Body fat distribution and risk of premenopausal breast cancer in the Nurses' Health Study II.J Natl Cancer Inst 2011;103:273-8.

7. Rock CL, Demark-Wahnefried W. Nutrition and survival after the diagnosis of breast cancer: a review of the evidence. J Clin Oncol 2002;20:3302-16. 
8. Pickhardt PJ, Jee $\mathrm{Y}, \mathrm{O}^{\prime}$ Connor SD, et al. Visceral adiposity and hepatic steatosis at abdominal CT: association with the metabolic syndrome. AJR Am J Roentgenol 2012;198:1100-7.

9. Camhi SM, Bray GA, Bouchard C, et al. The relationship of waist circumference and BMI to visceral, subcutaneous, and total body fat: sex and race differences. Obesity (Silver Spring) 2011;19:402-8.

10. Heymsfield SB, Shen W. Obesity: BAI as a new measure of adiposity--throw away your scale? Nat Rev Endocrinol 2011;7:321-2.

11. Hamilton EJ, Gianatti E, Strauss BJ, et al. Increase in visceral and subcutaneous abdominal fat in men with prostate cancer treated with androgen deprivation therapy. Clin Endocrinol (Oxf) 2011;74:377-83.

12. Renehan AG, Zwahlen M, Egger M. Adiposity and cancer risk: new mechanistic insights from epidemiology. Nat Rev Cancer 2015;15:484-98.

13. van Gemert WA, Monninkhof EM, May AM, et al. Association between changes in fat distribution and biomarkers for breast cancer. Endocr Relat Cancer 2017;24:297-305.

14. Picon-Ruiz M, Morata-Tarifa C, Valle-Goffin JJ, et al. Obesity and adverse breast cancer risk and outcome: Mechanistic insights and strategies for intervention. CA Cancer J Clin 2017;67:378-97.

15. Chen GC, Chen SJ, Zhang R, et al. Central obesity and risks of pre- and postmenopausal breast cancer: a doseresponse meta-analysis of prospective studies. Obes Rev 2016;17:1167-77.

16. Boonyaratanakornkit V, Pateetin $P$. The role of ovarian sex steroids in metabolic homestasis, obesity, and postmenopausal breast cancer: molecular mechanisms and therapeutic implications. Biomed Res Int 2015;2015:140196.

17. Rose DP, Vona-Davis L. Influence of obesity on breast cancer receptor status and prognosis. Expert Rev Anticancer Ther 2009;9:1091-101.

18. Collignon J, Lousberg L, Schroeder H, et al. Triplenegative breast cancer: treatment challenges and solutions. Breast Cancer (Dove Med Press) 2016;8:93-107.

19. Vona-Davis L, Howard-McNatt M, Rose DP. Adiposity, type 2 diabetes and the metabolic syndrome in breast cancer. Obes Rev 2007;8:395-08.

20. Deluche E, Leobon S, Desport JC, et al. Impact of body composition on outcome in patients with early breast cancer. Support Care Cancer 2018;26:861-8.
21. Klein S, Allison DB, Heymsfield SB, et al. Waist Circumference and Cardiometabolic Risk: a Consensus Statement from Shaping America's Health: Association for Weight Management and Obesity Prevention; NAASO, the Obesity Society; the American Society for Nutrition; and the American Diabetes Association. Obesity (Silver Spring) 2007;15:1061-7.

22. Vucenik I, Stains JP. Obesity and cancer risk: evidence, mechanisms, and recommendations. Ann N Y Acad Sci 2012;1271:37-43.

23. Jiang HS, Kuang XY, Sun WL, et al. Androgen receptor expression predicts different clinical outcomes for breast cancer patients stratified by hormone receptor status. Oncotarget 2016;7:41285-93.

24. Dunnwald LK, Rossing MA, Li CI. Hormone receptor status, tumor characteristics, and prognosis: a prospective cohort of breast cancer patients. Breast Cancer Res 2007;9:R6.

25. Slamon D, Eiermann W, Robert N, et al. Adjuvanttrastuzumab in HER2-positive breast cancer. N Engl J Med 2011;365:1273-83.

26. Perez EA, Patel T, Moreno-Aspitia A. Efficacy of ixabepilone in ER/PR/HER2-negative (triple-negative) breast cancer. Breast Cancer Res Treat 2010;121:261-71.

27. Bae SY, Kim S, Lee JH, et al. Poor prognosis of single hormone receptor-positive breast cancer: similar outcome as triple negative breast cancer. BMC Cancer 2015;18;15:138.

28. Gwak JM, Jang MH, Kim DI, et al. Prognostic value of tumor-associated macrophages according to histologic locations and hormone receptor status in breast cancer. Plos One 2015;10:e0125728.

29. Tiwari P, Blank A, Cui C, Schoenfelt KQ, et al. Metabolically activated macrophages in mammary adipose tissue link obesity to triple-negative breast cancer. J Exp Med 2019;216:1345-58.

30. Soguel L, Durocher F, Tchernof A, et al. Adiposity, breast density, and breast cancer risk: epidemiological and biological considerations. Eur J Cancer Prev 2017;26:511-20.

Cite this article as: Kim MS, Choi YJ, Lee YH. Visceral fat measured by computed tomography and the risk of breast cancer. Transl Cancer Res 2019;8(5):1939-1949. doi: 10.21037/ tcr.2019.09.16 